EUROPEAN ORGANIZATION FOR NUCLEAR RESEARCH
CERN - AB DEPARTMENT

CERN-AB-2005-086

CLIC Note 643

\title{
30 GHz High Power Production for CLIC
}

\author{
I. Syratchev
}

\begin{abstract}
The CLIC Power Extraction and Transfer Structure (PETS) is a passive microwave device in which bunches of the drive beam interact with the impedance of the periodically loaded waveguide and excite preferentially the synchronous $T M_{01}$ mode at $30 \mathrm{GHz}$. The RF power produced (several hundred MW) is collected at the downstream end of the structure by means of the Power Extractor and conveyed to the main linac structure. The PETS geometry is a result of multiple compromises between beam stability along a single decelerator sector $(600 \mathrm{~m})$ and the active length of the structure to match the main linac RF power needs and layout. Surface electric and magnetic fields, power extraction method, HOM damping, ON/OFF capability and fabrication technology were all evaluated to provide a reliable design.
\end{abstract}

Presented at the $7^{\text {th }}$ International High Energy Density and High Power RF Workshop, Kalamata, Greece, 13-17 ${ }^{\text {th }}$ June 2005

Geneva, Switzerland

November 2005 


\title{
$30 \mathrm{GHz}$ high power production for CLIC
}

\author{
I. Syratchev
}

CERN, Geneva, Switzerland

\begin{abstract}
The CLIC Power Extraction and Transfer Structure (PETS) is a passive microwave device in which bunches of the drive beam interact with the impedance of the periodically loaded waveguide and excite preferentially the synchronous $T M_{01}$ mode at $30 \mathrm{GHz}$. The RF power produced (several hundred MW) is collected at the downstream end of the structure by means of the Power Extractor and conveyed to the main linac structure. The PETS geometry is a result of multiple compromises between beam stability along a single decelerator sector $(600 \mathrm{~m})$ and the active length of the structure to match the main linac RF power needs and layout. Surface electric and magnetic fields, power extraction method, HOM damping, ON/OFF capability and fabrication technology were all evaluated to provide a reliable design.
\end{abstract}

Keywords: Enter Keywords here.

PACS: 29.27.Bd, 41.75.Lx.

\section{THE CLIC POWER EXTRACTION AND TRANSFER STRUCTURE (PETS).}

The CLIC Power Extraction and Transfer Structure (PETS) is a passive microwave device in which bunches of the drive beam interact with the impedance of the periodically corrugated waveguide and excite preferentially the synchronous TM01 mode at $30 \mathrm{GHz}$. In the process the beam kinetic energy is converted into electromagnetic energy at the mode frequency, which travels along the structure with the mode group velocity [1]. The RF power produced is collected at the downstream end of the structure by means of the Power Extractor and conveyed to the main linac structure by means of rectangular waveguides.

The choice of PETS geometry is a result of multiple compromises between the drive beam stability along the whole decelerator sector $(\sim 600 \mathrm{~m})$ and the active length of the PETS which is given by the main linac RF power needs and the layout. Surface electric field, power extraction method, HOM damping, ability to cancel RF power generation if needed, and fabrication technology were all evaluated to ensure a reliable design. The final PETS design consists of a $22.5 \mathrm{~mm}$ diameter circular waveguide with shallow ( $\sim 1.3 \mathrm{~mm}$ deep) sinus-type corrugations with 140 degrees phase advance per period $(3.8885 \mathrm{~mm})$. Eight HOM damping slots are placed symmetrically around the circumference splitting the whole structure into 8 identical pieces. To simplify the fabrication, the active profile of each of the 8 racks was chosen to be flat, see Figure 1 . The damping slot width $(2 \mathrm{~mm})$ and the slot's rounding radii $(0.8 \mathrm{~mm})$ provide a quasi-constant surface electric field distribution with a maximum of about $120 \mathrm{MV} / \mathrm{m}$ at the nominal RF power level. In this configuration the $600 \mathrm{~mm}$ PETS active length is sufficient to extract $642 \mathrm{MW}$ of RF power from the $181 \mathrm{~A}, 15 \mathrm{GHz}$ drive beam. 
Assuming an extraction and transfer efficiency of $94 \%$ this is enough to deliver 151 MW to each of the four CLIC accelerating structures. The CLIC PETS parameters are shown in Table 1.

TABLE 1. The CLIC PETS parameters

\begin{tabular}{lc}
\hline Synchronous frequency, $\mathrm{GHz}$ & 29.9855 \\
Aperture, mm & 22.5 \\
$\mathrm{R} / \mathrm{Q}, \Omega / \mathrm{m}$ & 320 \\
Group velocity / C & 0.789 \\
Phase advance/cell, degrees & 140 \\
Active length, m & 0.6 \\
Drive beam current, A & 181 \\
RF power generated, MW & 642 \\
Surface electric field, MV/m & 120 \\
Extraction and transfer efficiency, $\%$ & 94 \\
Damping slots & $8 \times 2 \mathrm{~mm}$ \\
\hline
\end{tabular}

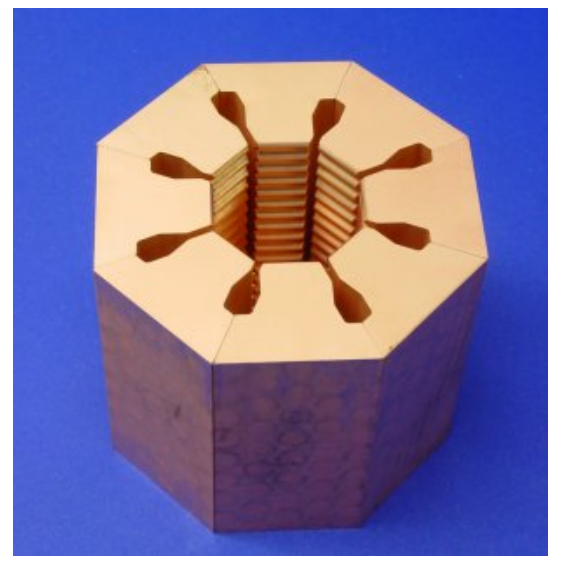

FIGURE 1. The PETS machining test prototype.

\section{PETS RF Power Extractor}

The extraction of RF power from over-moded waveguides is not a trivial task, especially when one needs to maintain the beam aperture. To make it efficient and compact a new broad-band 8-channel quasi-optical extraction coupler based on the multimode mixing approach has been designed to couple out the power from the CLIC PETS with an efficiency of $99.6 \%$ according to the HFSS simulations [2]. The latest version of the coupler consists of two parts, a mode launcher, and a diffraction / extraction section; these units provide an efficient step-by-step conversion of the energy from the $\mathrm{E}_{01}$ mode of the over-moded circular waveguide to the fundamental $\mathrm{H}_{10}$ mode of the eight radial standard rectangular waveguides. In turn these eight waveguides are combined in pairs with further $\mathrm{H}_{20}$ to $\mathrm{H}_{01}$ mode conversion, followed by four circular waveguides with reduced losses, as shown in Figure 2. The total length of the extraction is $70 \mathrm{~mm}$. A low power prototype has been built and RF tested and shown to be in good (within 1\%) agreement with the HFSS simulation, see Figure 3. Since the PETS is very over-moded, any geometrical perturbation will provoke coupling of the decelerating mode into unwanted HOMs. As 
a result, a long adiabatic matching section with a number of gradually reduced corrugations between the active PETS part and the power extractor is needed. The matching section has a total length of $58 \mathrm{~mm}$ (15 periods) and has a reflection and mode conversion expected to be better than $-40 \mathrm{~dB}$. Finally the total length of the PETS including all elements is $770 \mathrm{~mm}$.
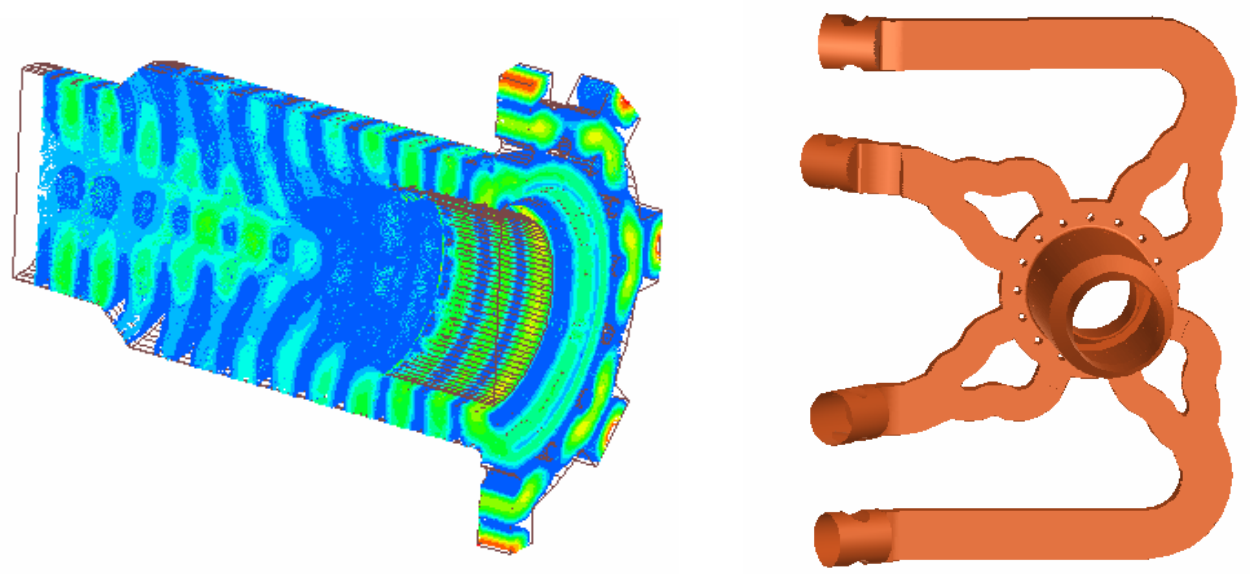

FIGURE 2. Electric field plot in the coupler volume as simulated with HFSS (left) and artistic view of the extractor head (right).
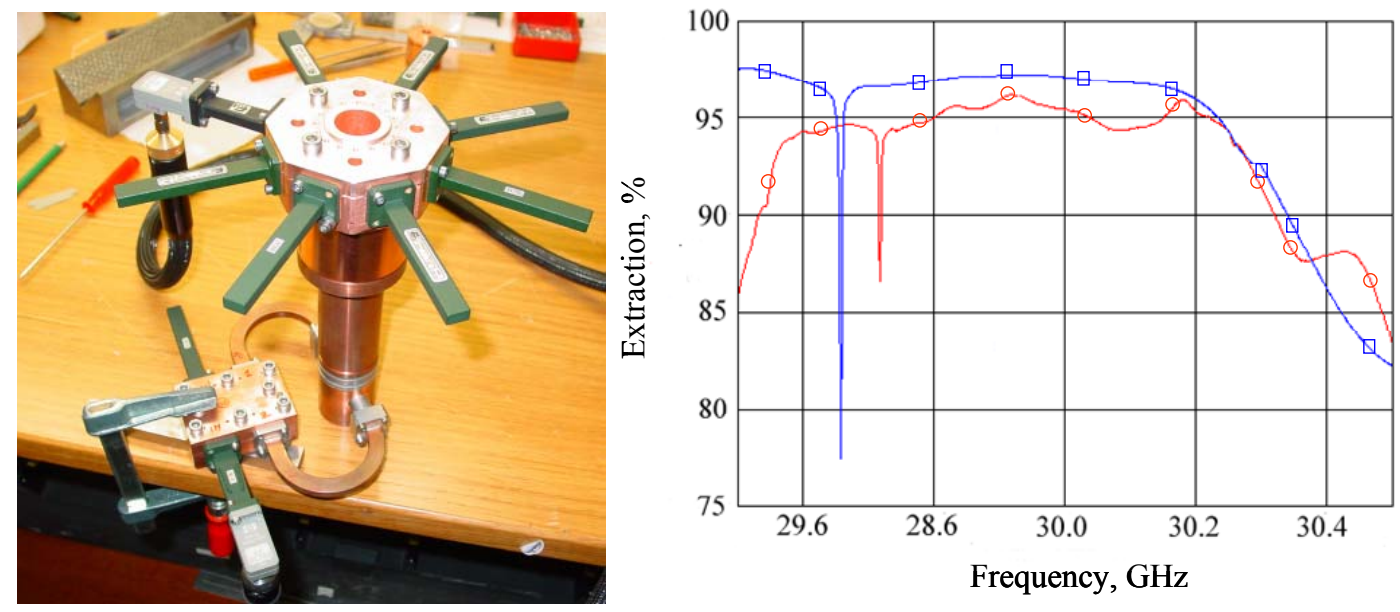

FIGURE 3. The PETS RF power coupler low power prototype test set-up (left). Extraction efficiency (right) - measurement (circles) and simulation (squares).

\section{Transverse Higher Order Mode (HOM) damping and detuning}

To avoid drive beam losses along the decelerator, the strong suppression of transverse HOMs is absolutely necessary. The most dangerous transverse mode has a frequency and group velocity practically identical to the decelerating one. The only way to damp it is to use its symmetry properties. The damping mechanism of this mode can be explained as a coherent radiation of many RF sources represented by the individual period of the corrugations into eight infinite radial slots. 
The angle of radiation here depends on the phase advance and the distance between periods. The higher the phase advance, the smaller the angle and the less the damping. In any case the radiation (damping) is strongest when the phase advance and period are matched in frequency. For practical reasons the infinite slot is replaced by a broadband RF matched load. The iterative optimization of the slot configuration and the damping was done using HFSS [3], GDFIDL [4] was used to verify the whole structure behavior in the time domain. Finally beam dynamic simulations with PLACET [5] were used to confirm stable beam transportation along the decelerator.

It was found, that in the presence of the damping slots, the lowest transverse mode splits into two different frequency modes with different phase relations between slots and aperture. This brings extra benefits due to the naturally introduced quasi-detuning and hence stronger suppression of the wake at shorter distances. In addition the depth of the regular part of the slots was tuned in pairs of 4 to provide the best damping of each of the two modes. The parameters of the modes whose total contribution to the wake amplitude is more than $95 \%$ were calculated using HFSS and are shown in Table 2 .

TABLE 2. The transverse modes in PETS.

\begin{tabular}{lcccc}
\hline Parameter & Mode 1 & Mode 2 & Mode 3 & Mode 4 \\
\hline $\mathrm{W}_{\mathrm{T}} /(1-\beta), \mathrm{V} / \mathrm{pC} / \mathrm{m} / \mathrm{mm}$ & 2.144 & 0.874 & 0.04 & 0.109 \\
Frequency, GHz & 28.031 & 35.092 & 41.674 & 46.272 \\
$\mathrm{Q}_{\mathrm{LOADED}}$ & 44.1 & 39.8 & 575 & 1387 \\
$\mathrm{~V}_{\text {GROUP }} / C$ & 0.888 & 0.651 & -0.078 & 0.09 \\
\hline
\end{tabular}

Beam dynamic simulations have shown that an insufficiently damped HOM of the higher bands (see Table 2, Mode 4) can be troublesome due to its low group velocity, even though the impedance is quite small [6]. To avoid this, frequency detuning was introduced using a linear increase of the phase advance per cell (from 132 to 140 degrees) along the structure, while keeping the aperture constant. This resulted in an effective suppression of the higher band HOM by a factor of ten. The final envelops of the transverse wake and impedance as simulated with GDFIDL for the full structure length and a single bunch sigma equal to $0.7 \mathrm{~mm}$ are shown in Figure 4.
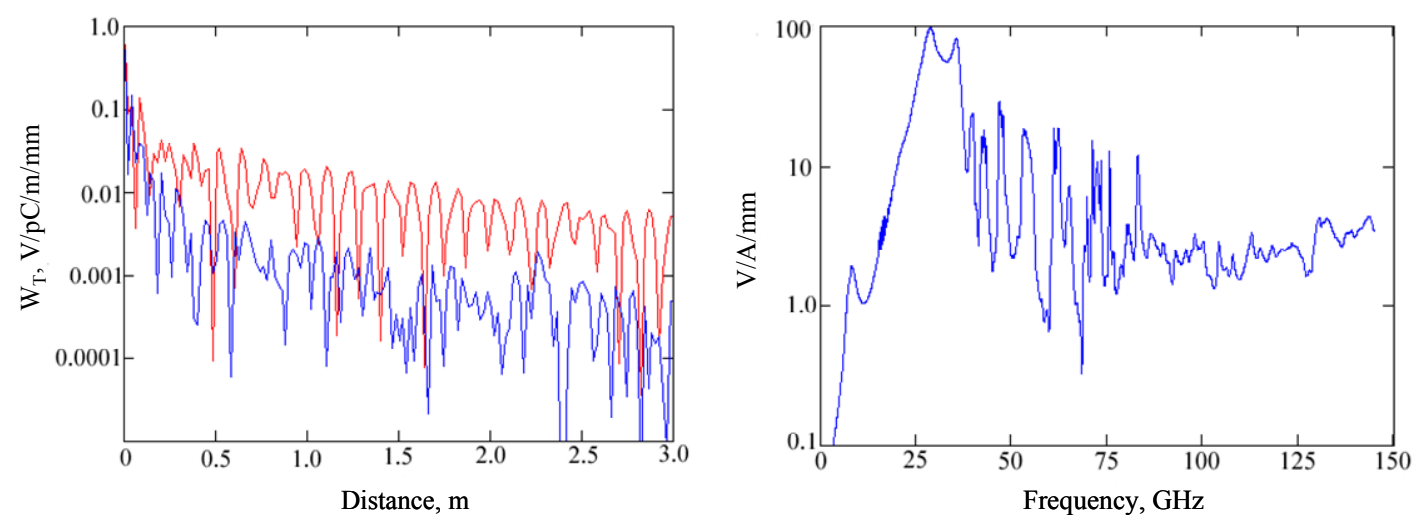

FIGURE 4. The PETS transverse wake envelopes with damping (upper, left), with damping and detuning (lower, left) and impedance with damping and detuning (right). 
The envelopes of a $3 \sigma$ beam with an initial offset of $\Delta Y=0.3 \sigma_{y}$ along the decelerator with and without transverse wakes simulated with PLACET are shown in Figure 5. One can see that the transverse HOM have now practically no effect on the beam losses.

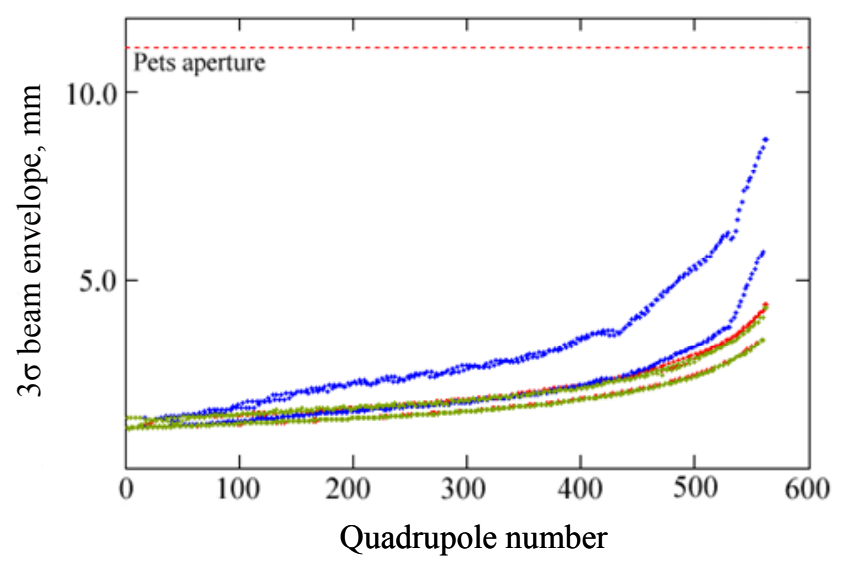

FIGURE 5. The envelopes of a $3 \sigma$ beam with an initial offset of $\Delta \mathrm{Y}=0.3 \sigma_{\mathrm{y}}$ along the decelerator with damping (blue), with damping and detuning (brown) and without transverse wakes effects (red).

\section{Local Termination of the RF Power Production (ON/OFF Option).}

During machine operation it will be necessary to locally turn the RF power production OFF when either a PETS or an accelerating structure fails due to breakdown. It can be shown that the RF power generated by the beam at the end of the constant impedance structure will be zero if the synchronous frequency is detuned by an amount $\pm \beta C /(1-\beta) L$, where $\beta-\mathrm{V}_{\text {group }} / C$ and $L-$ length of the structure. In this case this corresponds to about $2 \mathrm{GHz}$ frequency detuning. It has been found that such a strong detuning can be achieved by inserting 4 thin $(1.6 \mathrm{~mm})$ wedges through 4 of the 8 damping slots in such a way that the edge position of the wedge does not exceed the beam aperture, see Fig. 6. The wedge geometry was modified in a way that an an intermediate wedge position there is no electrical field enhancement in the $0.2 \mathrm{~mm}$ gap between the wedge and the wall, nor any RF power leakage into the HOM loads [7]. Thus the device can operate as a variable attenuator.
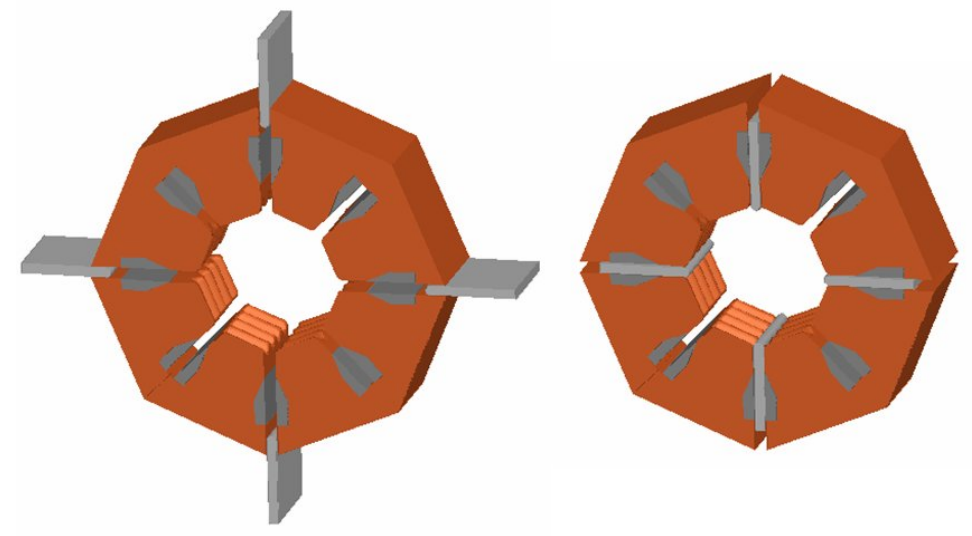

FIGURE 6. PETS ON (left) and OFF (right) configurations with detuning wedges 
The full PETS geometry including matching section and output coupler was simulated with GDFIDL to verify the performance and potential problems. The reconstruction of the output RF power build-up using a single bunch response, made it possible to reproduce realistic pulse envelopes and to demonstrate numerically the ON/OFF capability, see Figure 7.
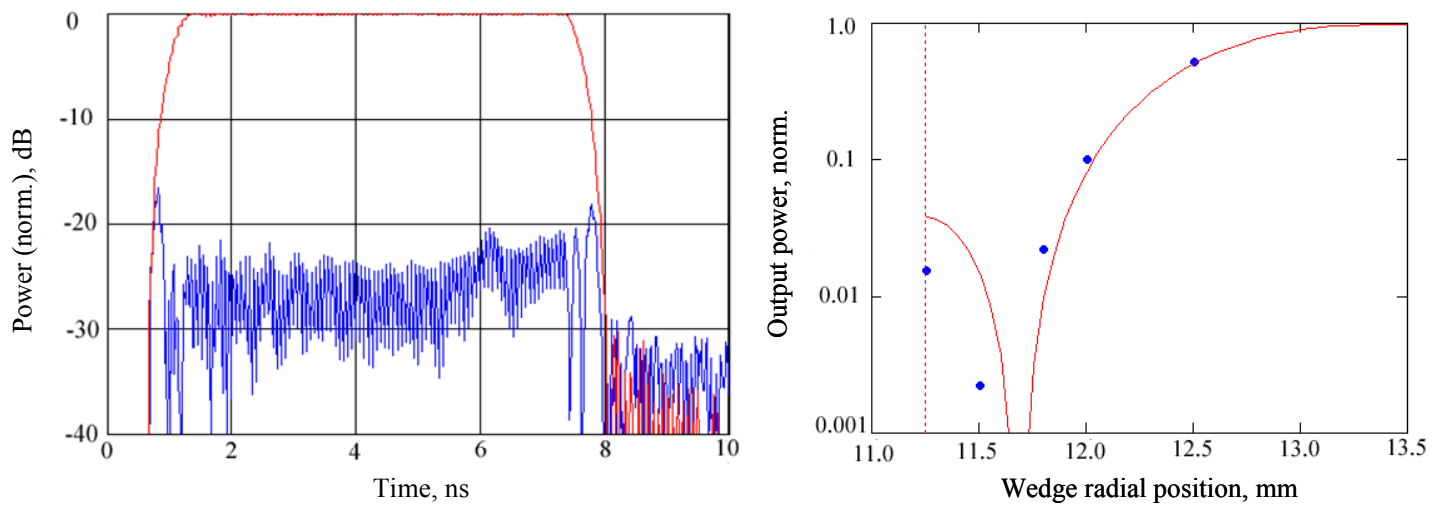

FIGURE 7. The reconstructed pulse envelopes for the two regimes of PETS operation (left). The output power level versus wedge edge radial position as reconstructed from GDFIDL (dots) and calculated analytically using HFSS data (line).

\section{FUTURE PLANS}

In the coming years, 2005-2009, the CLIC Test Facility (CTF3) will be built and operated at CERN to verify the key issues of the CLIC scheme. The demonstration of the nominal operation of the CLIC PETS is one of them. Since the CTF3 drive beam current is 5 times smaller than the nominal CLIC current, this is challenging and will involve further studies, developments and industrialization efforts.

\section{REFERENCES}

1. The CLIC Study Team, Ed. G. Guignard, "A $3 \mathrm{TeV} \mathrm{e}^{+} \mathrm{e}^{-}$Linear Collider Based on CLIC Technology", Report CERN 2000-008, July 2000.

2. I. Syratchev, "Efficient RF Power Extraction from the CLIC Power Extraction and Transfer Structure (PETS)", CLIC Note 571, 2003

3. HFSS, http//www.ansoft.com/products/hf/hfss/.

4. W. Bruns, I. Syratchev, "Time Domain Simulations of the CLIC PETS with GDFIDL", PAC20001, Chicago, June 2001 (CN 490).

5. D. Schulte, CERN/PS 2000-028 (AE 2000)

6. D. Schulte and I. Syratchev "Considerations on the Design of the Decelerator of the CLIC Test Facility (CTF3)”, PAC2005, May 2005.

7. I. Syratchev "The CLIC Power Extraction and Transfer Structure (PETS)", CLIC Meeting 03.12.2004, http://clic-meeting.web.cern.ch/clic-meeting/2004/12_03min.html. 